\title{
The bursting nature of star formation in compact star-forming galaxies from the Sloan Digital Sky Survey
}

\author{
Y. I. Izotov ${ }^{1,2},{ }^{\star}$ N. G. Guseva ${ }^{1,2}$, K. J. Fricke ${ }^{2,3}$ \& C. Henkel ${ }^{2,4}$ \\ ${ }^{1}$ Main Astronomical Observatory, Ukrainian National Academy of Sciences, 27 Zabolotnoho str., Kyiv 03680, Ukraine \\ ${ }^{2}$ Max-Planck-Institut für Radioastronomie, Auf dem Hügel 69, 53121 Bonn, Germany \\ ${ }^{3}$ Institut für Astrophysik, Göttingen Universität, Friedrich-Hund-Platz 1, 37077 Göttingen, Germany \\ ${ }^{4}$ Astronomy Department, King Abdulaziz University, P.O. Box 80203, Jeddah 21589, Saudi Arabia
}

Accepted XXX. Received YYY; in original form ZZZ

\begin{abstract}
We study integrated characteristics of $\sim 14000$ low-redshift $(0<z<1)$ compact star-forming galaxies (SFGs) selected from the Data Release 12 of the Sloan Digital Sky Survey. It is found that emission of these galaxies is dominated by strong young bursts of star formation, implying that their luminosities experience rapid variations on a time scale of a few Myr. Reducing integrated characteristics of these galaxies to zero burst age would result in a considerably tighter and almost linear relation between stellar mass and star formation rate (SFR). The same correction implies that the specific star formation rate (the ratio of SFR and stellar mass) is not dependent on the galaxy stellar mass. We conclude that the correction for rapid luminosity evolution must be taken into account in a similar way when comparing different samples of lowand high-redshift SFGs. If the bursting nature of star formation and young burst ages are characteristics of the galaxies selected at high redshifts, the age correction of observed SFRs derived from the $\mathrm{H} \beta$ emission line or UV continua would modify the derived SFR densities in the early universe.
\end{abstract}

Key words: galaxies: dwarf — galaxies: fundamental parameters — galaxies: irregular — galaxies: ISM — galaxies: starburst

\section{INTRODUCTION}

Relations between luminosities, stellar masses, and star formation rates (SFRs) of star-forming galaxies (SFGs) and their variations with redshift play an important role in our understanding of their formation and evolution on cosmological time scales.

These relations for various samples of low- $z$ and high- $z$ galaxies were studied in numerous papers (e.g. Tremonti et al. 2004; Maiolino et al. 2008; Manucci et al. 2010; Steidel et al. 2014; Izotov et al. 2011, 2015). For example, Maiolino et al. (2008) found an evolution of the stellar mass-metallicity relation with redshift in the sense that high$z(z \sim 3.5)$ galaxies are more metal-poor compared to low- $z$ galaxies for the same stellar mass. This effect is more pronounced for low-mass galaxies. Manucci et al. (2010) considered a more general relation between stellar mass $M_{\star}$, metallicity, and SFR. They found that high- $z$ galaxies are characterised by progressively higher SFRs.

On the other hand, there is some evidence that properties of low- $z$ "Green Pea" (GP) galaxies and Luminous

* E-mail: izotov@mao.kiev.ua
Compact Galaxies (LCGs) and high- $z$ Lyman-alpha emitting (LAE) and Lyman-break galaxies (LBG) are similar with respect to the stellar mass, SFR and metallicity (Cardamone et al. 2009; Izotov et al. 2011, 2015). It was found by Izotov et al. (2014) and Izotov et al. (2015) that luminosity-metallicity, mass-metallicity and mass-SFR relations for Sloan Digital Sky Survey (SDSS) compact SFGs are much flatter than e.g. the Tremonti et al. (2004) relations for the main sequence low- $z$ SFGs. They also found that low- $z$ galaxies with high SFRs do not deviate from the relation established for galaxies with lower SFRs. Furthermore, no considerable redshift dependence was found.

These results indicate inconsistencies between relations obtained in different studies. Among possible sources of inconsistencies are different criteria for the sample selection and a variety of strong-line methods, which are used for the metallicity determination. Element abundances derived by different methods can be different by more than 0.5 dex.

Furthermore, it is generally assumed that star formation in SFGs occurs continuously at time scales of $~ 100$ Myr either with a constant or an exponentially decreasing SFR. However, the possibility that star formation may occur in strong bursts with rapidly changing $\mathrm{H} \beta$ line and UV contin- 
uum luminosities by a factor of several times on time scales of $\leqslant 10 \mathrm{Myr}$ is rarely taken into account (Izotov et al. 2014, 2015). The presence of bursts may only slightly change the deduced total stellar masses, which are determined from the galaxy continuum emission in the visible and longer wavelength ranges, but may significantly affect the $\mathrm{H} \beta$ emission line and UV luminosities and hence SFRs derived from these luminosities. As a consequence, the comparison of bursting SFGs in different samples should be done only after reduction of their luminosities to the same burst age (e.g. Izotov et al. 2015).

In this paper we consider an impact of star formation history on the $\mathrm{H} \beta$ line and UV continuum luminosities and present evidence that star formation in compact SFGs has a bursting nature. In Sect. 2 we discuss the sample. The technique used for the determination of luminosities and stellar masses is described in Sect. 3. Evidence for the bursting nature of star formation in SDSS compact SFGs is discussed in Sect. 4. The relations stellar mass - SFR and stellar mass - specific SFR (sSFR) are considered in Sect. 5. The main results of the paper are summarised in Sect. 6 .

\section{THE SAMPLE OF COMPACT STAR-FORMING GALAXIES}

\subsection{Selection criteria}

The SDSS Data Release 12 (DR12) (Alam et al. 2015) was used to select a sample of compact SFGs applying selection criteria by Izotov et al. (2015): 1) the angular galaxy radius on the SDSS images is $R_{50} \leqslant 3$ arcsec, where $R_{50}$ is the galaxy's Petrosian radius within which $50 \%$ of the galaxy's flux in the SDSS $r$ band is contained; 2) H II regions in spiral galaxies were excluded using SDSS images; 3) galaxies with AGN activity were excluded using line ratios. Applying these criteria, $\sim 14000$ galaxies with redshifts $z<1$ were selected.

The location of the selected galaxies in the $\left[\begin{array}{ll}\mathrm{O} & \text { III }\end{array}\right] 25007 / \mathrm{H} \beta-\left[\begin{array}{ll}\mathrm{N} & \text { II }\end{array}\right] \lambda 6584 / \mathrm{H} \alpha$ diagnostic diagram ( Baldwin, Phillips \& Terlevich 1981) is shown in Fig. 1. The solid line by Kauffmann et al. (2003) separates SFGs and active galactic nuclei (AGN). Our sample galaxies are located in the SFG region and thus their interstellar medium is ionized by hot stars in the star-forming regions.

The distribution of $R_{50}$ for our galaxies with averaged value of $\sim 1$ arcsec is presented in Fig. 2a. These small angular sizes indicate that a considerable fraction of the galaxy light is concentrated inside the spectroscopic aperture. This results in relatively small aperture corrections with average value $\sim 0.6 \mathrm{mag}$ to convert the fluxes derived inside the spectroscopic aperture to the total galaxy fluxes (Fig. 2b). Furthermore, small angular sizes also indicate that most of the selected galaxies are unresolved or barely resolved on the SDSS images. True angular sizes can be much smaller. This was demonstrated e.g. by Izotov et al. (2016b) who found angular radii $R_{50} \lesssim 0.1$ arcsec for five compact SFGs at redshifts $\sim 0.3$. All this implies that our galaxies have small linear radii of a few kpc. We note that the adopted upper limit $R_{50}=3$ arcsec is somewhat arbitrary. It is two and three times higher than the radii of spectroscopic apertures used in the SDSS-II and SDSS-III surveys, respectively. Reducing $R_{50}$ to the radii of spectroscopic apertures will not influence the selection of distant SFGs with $z \gtrsim 0.3$, but many

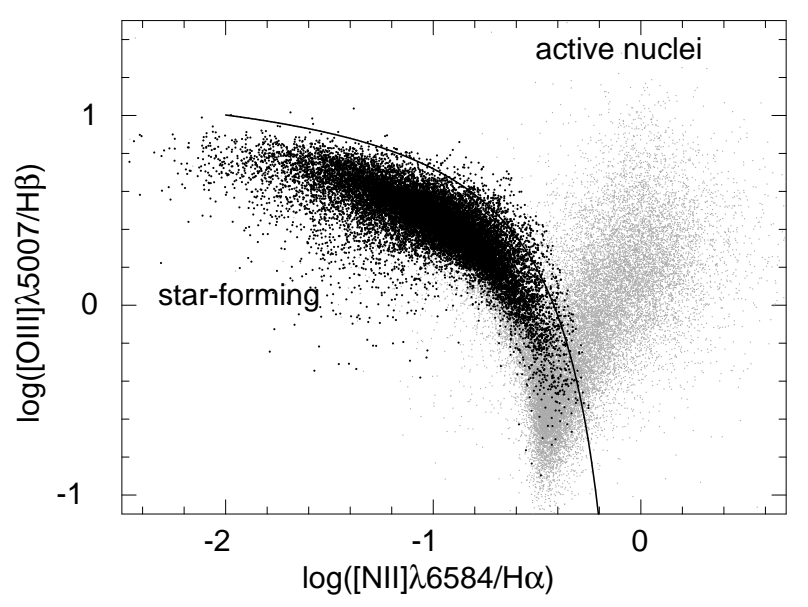

Figure 1. The Baldwin-Phillips-Terlevich (BPT) diagram (Baldwin et al. 1981) for narrow emission-line galaxies. The compact star-forming galaxies (this paper) are represented by black dots. Also, plotted are emission-line galaxies from SDSS DR7 (cloud of grey dots). The solid line by Kauffmann et al. (2003) separates star-forming galaxies (SFG) from active galactic nuclei (AGN).

lower-redshift SFGs with similar properties will be missed. Therefore, for the purpose of this paper the criterion $R_{50} \leqslant$ 3 arcsec is optimal.

The location of our selected SFGs on the SDSS $(g-$ $r)-(r-i)$ colour-colour diagram is represented in Fig. 3 by black symbols. This diagram was used by Cardamone et al. (2009) to select GPs with strong emission lines at redshifts $z \sim 0.13-0.3$. They are located in the upper left part of the diagram in the region with $(r-i) \lesssim-0.5 \mathrm{mag}(g-r)$ $\gtrsim 0.5$ mag. Our compact SFGs are distributed in a similar way as the LCGs by Izotov et al. (2011) (their fig. 4). The galaxies with high $\mathrm{EW}(\mathrm{H} \beta) \gtrsim 100 \AA$ are distributed in a very wide range of the $(r-i)$ colours, which is determined by the observed wavelength of the strong redshifted $[\mathrm{O}$ III $] \lambda 5007 \AA$ emission line. Galaxies at redshifts of $\sim 0.2-0.3$ are located in the upper left corner of the diagram, while galaxies at $z \sim$ 0.5 are in the lower right corner with $(r-i) \sim 0.5-1.0$. The distribution of our SFGs with low $\operatorname{EW}(\mathrm{H} \beta)<50 \AA$ is more compact because of the much weaker $[\mathrm{O}$ III $] \lambda 5007 \AA$ emission. Most of these SFGs are located in the $(r-i)$ range $0.0-$ 0.5 and are coincident with the location of the SDSS QSOs with blue continua, as it was shown by Izotov et al. (2011). On the other hand, compact SFGs are clearly different from the SDSS "normal" quiescent galaxies (dense cloud of grey symbols with $(r-i) \sim 0.4-0.6 \mathrm{mag}$ and $(g-r)>0.6 \mathrm{mag})$, which have redder $(g-r)$ colour.

The SDSS photometric and spectroscopic data in combination with the Galaxy Evolution Explorer (GALEX) photometric data are used in Section 3 to derive galaxy stellar masses $M_{\star}, \mathrm{H} \beta$ luminosities $L(\mathrm{H} \beta)$, far-UV (FUV) and nearUV (NUV) luminosities, SFRs and sSFRs.

Selected galaxies are distributed over a redshift range of $0-1$ with average value $z \sim 0.21$ (Fig. 4a). The distributions of integrated characteristics of these galaxies $\mathrm{EW}(\mathrm{H} \beta), L(\mathrm{H} \beta), M_{\star}$ are shown in Figs. $4 \mathrm{~b}-4 \mathrm{~d}$. They closely resemble the properties of high- $z$ SFGs with strong emission lines (Izotov et al. 2014, 2015), making them 

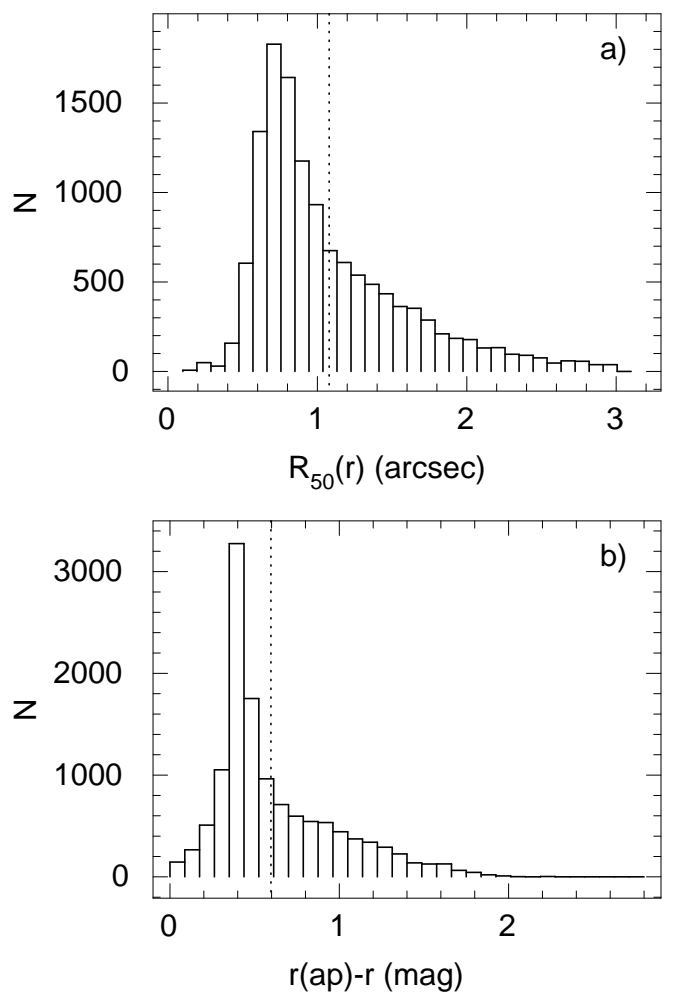

Figure 2. Distributions in the SDSS $r$ band of (a) Petrosian radii $R_{50}(r)$ and (b) aperture corrections for our compact SFGs. Vertical dotted lines in both panels indicate average values.

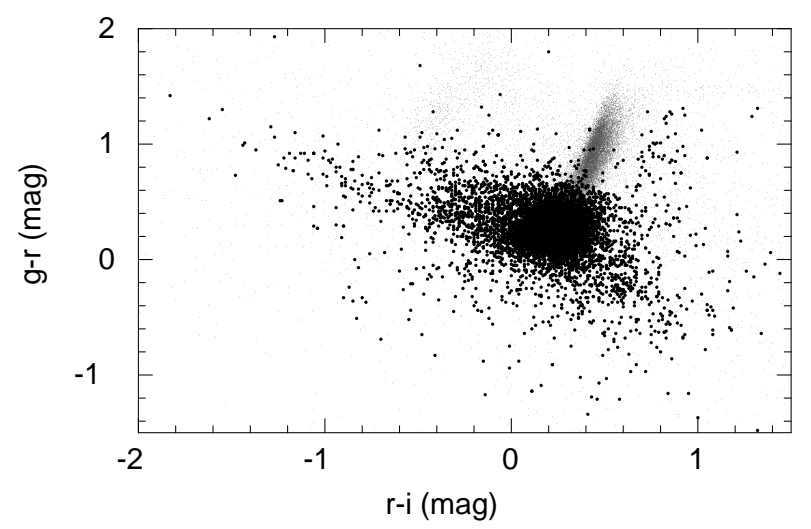

Figure 3. $(g-r)-(r-i)$ colour-colour diagram for compact SFGs (black symbols). For comparison, a representative sample of SDSS "normal" galaxies is shown by grey symbols.

the local counterparts of galaxies in the early universe. Izotov et al. (2015) showed that low- $z$ compact SFGs follow the same mass - metallicity, luminosity - metallicity and mass - SFR relations with high- $z$ emission-line galaxies at $z=2-11$ studied by Cullen et al. (2014), Steidel et al. (2014), Maiolino et al. (2008), Maier et al. (2014), Troncoso et al. (2014), Sanders et al. (2015), Salmon et al. (2015), Bouwens et al. (2012), Coe et al. (2013), and Oesch et al. (2014). They have similar SFRs $\sim$ $1-100 \mathrm{M}_{\odot} \mathrm{yr}^{-1}$ and overlapping range of stellar masses $\sim$ $10^{9}-10^{11} \mathrm{M}_{\odot}$. Furthermore, galaxies from our sample may
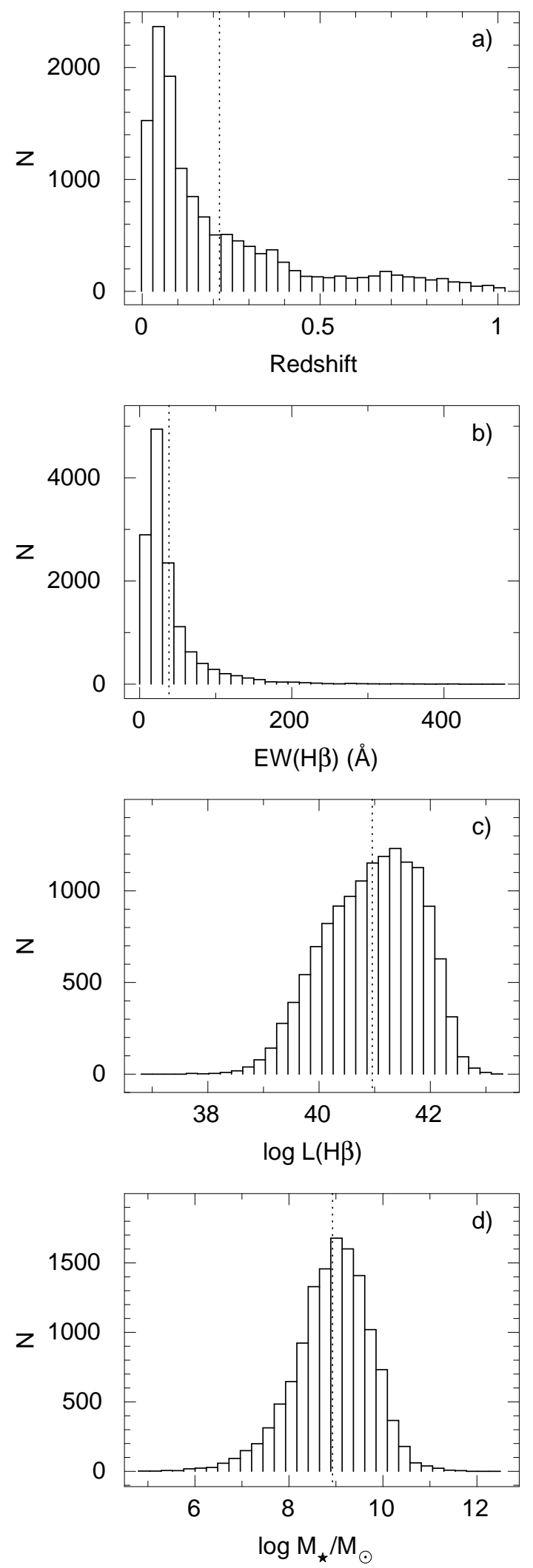

Figure 4. Distributions of compact star-forming galaxies on (a) redshift $z$, (b) rest-frame equivalent width $\mathrm{EW}(\mathrm{H} \beta)$ of the $\mathrm{H} \beta$ emission line, (c) aperture- and extinction-corrected luminosity $L(\mathrm{H} \beta)$ of the $\mathrm{H} \beta$ emission line expressed in $\operatorname{erg} \mathrm{s}^{-1}$, and (d) stellar galaxy mass $M_{\star}$. Vertical dotted lines indicate average values. 

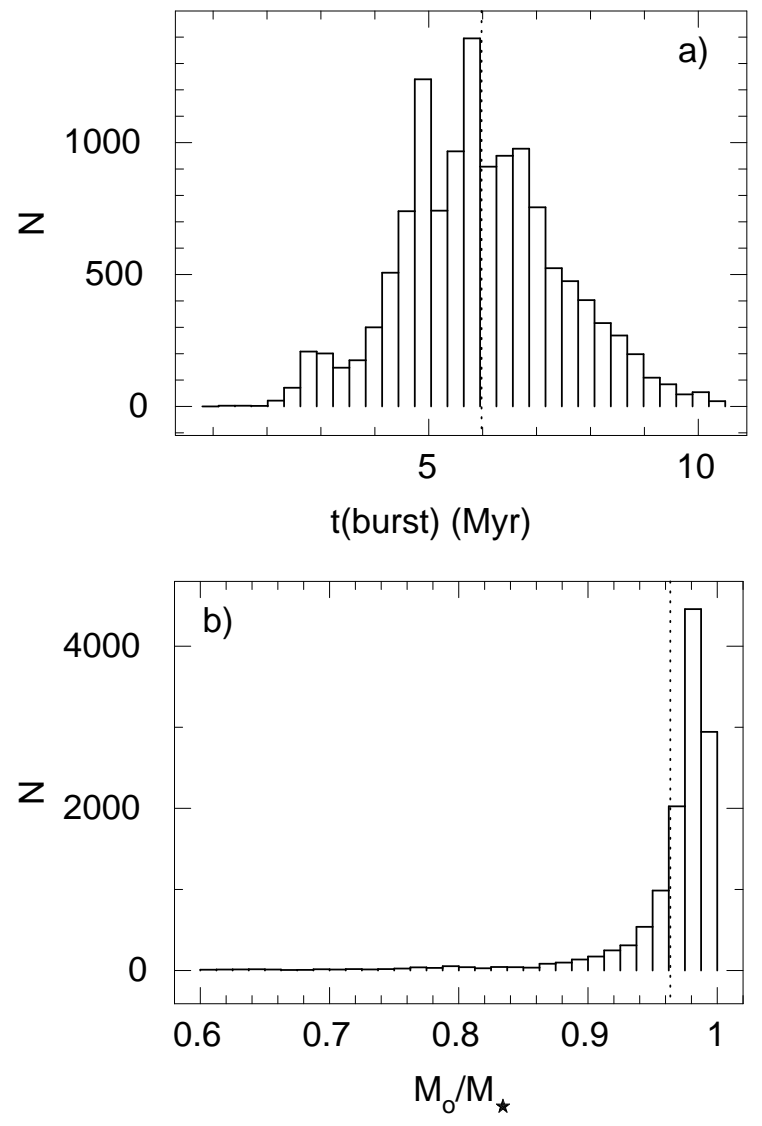

Figure 5. Distributions of compact SFGs on (a) starburst ages and (b) the fraction of the mass of the old stellar population. Vertical dotted lines indicate average values.

contain a significant fraction of Lyman continuum leaking galaxies (Izotov et al. 2016a,b). Their high- $z$ counterparts likely were the main sources of the universe reionization at redshifts $\sim 5-10$.

\section{THE DETERMINATION OF INTEGRATED PARAMETERS}

\subsection{Correction for extinction and aperture}

We measured emission-line fluxes and equivalent widths in the SDSS spectra using the IRAF $^{1}$ SPLOT routine. The observed decrement of several hydrogen Balmer emission lines was used to correct the line fluxes relative to the $\mathrm{H} \beta$ flux for two effects: (1) reddening adopting the extinction curve of Cardelli et al. (1989) and (2) underlying hydrogen stellar absorption that is derived simultaneously by an iterative procedure as described in Izotov et al. (1994).

The correction for reddening was done in two steps. First, the observed spectra, uncorrected for redshift, were corrected for the Milky Way extinction with $A(V)_{\mathrm{MW}}$ from

1 IRAF is the Image Reduction and Analysis Facility distributed by the National Optical Astronomy Observatory, which is operated by the Association of Universities for Research in Astronomy (AURA) under cooperative agreement with the National Science Foundation (NSF).
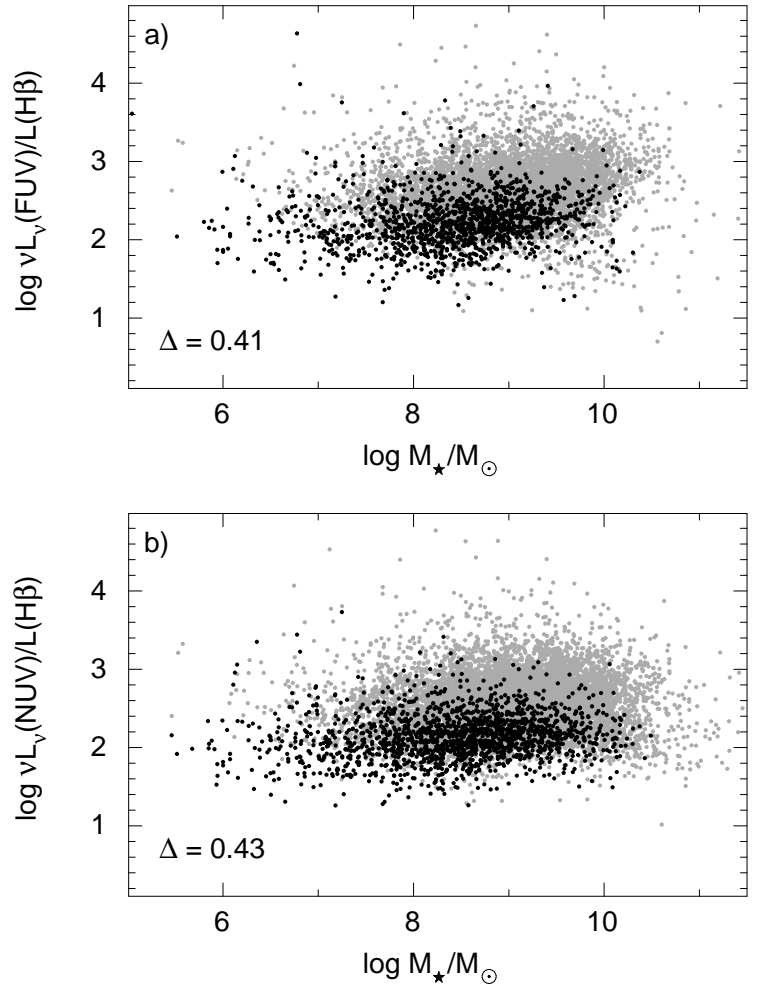

Figure 6. The dependence of the extinction-corrected FUVto- $\mathrm{H} \beta$ (a) and NUV-to- $\mathrm{H} \beta$ (b) luminosity ratios on the stellar masses. Additionally, $L(\mathrm{H} \beta)$ is corrected for the spectroscopic aperture. Galaxies with rest-frame $\mathrm{H} \beta$ equivalent widths $\mathrm{EW}(\mathrm{H} \beta)<100 \AA$ and $\mathrm{EW}(\mathrm{H} \beta) \geqslant 100 \AA$ are shown by grey and black dots, respectively. The values of $\Delta$ are the differences in log scale between the averaged values of the FUV-to-H $\beta$ and NUVto- $\mathrm{H} \beta$ luminosity ratios for galaxies with low and high $\mathrm{EW}(\mathrm{H} \beta)$ s.

the NASA Extragalactic Database $(\mathrm{NED})^{2}$ and $R(V)=$ $A(V) / E(B-V)=3.1$. Then, the rest-frame spectra were corrected for the internal extinction $A(V)$ int of the galaxies, obtained from the hydrogen Balmer decrement after its correction for the Milky Way extinction.

The same $A(V)_{\mathrm{MW}}, A(V)_{\mathrm{int}}$, and $R(V)$ were used to correct GALEX FUV and NUV fluxes.

In addition to extinction the $\mathrm{H} \beta$ fluxes were also corrected for the aperture using the relation $2.5^{r(\mathrm{ap})-r}$, where $r$ and $r$ (ap) are the SDSS $r$ total magnitude and the magnitude within the round spectroscopic aperture, respectively (Izotov et al. 2015). The aperture corrections of fluxes for most selected galaxies are small, less than a factor of 2 (see Fig. 2b). Only for less than $30 \%$ of the sample a larger aperture correction is needed.

2 The NASA/IPAC Extragalactic Database (NED) is operated by the Jet Propulsion Laboratory, California Institute of Technology, under contract with the National Aeronautics and Space Administration. 


\section{2 $\mathrm{H} \beta$ and UV luminosities and star formation rates}

The extinction- and aperture-corrected $\mathrm{H} \beta$ fluxes were transformed to luminosities adopting luminosity distances derived with the cosmological calculator (NED, Wright 2006), based on the cosmological parameters $H_{0}=67.1 \mathrm{~km}$ $\mathrm{s}^{-1} \mathrm{Mpc}^{-1}, \Omega_{\Lambda}=0.682, \Omega_{m}=0.318$ (Ade et al. 2014). Similarly, the GALEX FUV and NUV luminosities were derived from the extinction-corrected fluxes.

SFRs were obtained from the extinction- and aperturecorrected $\mathrm{H} \beta$ luminosities adopting the Kennicutt (1998) relation between SFR and $L(\mathrm{H} \alpha)$ and an $\mathrm{H} \alpha / \mathrm{H} \beta$ flux ratio of 2.8 .

\subsection{Stellar masses}

To fit the SEDs and to derive stellar masses we used the SDSS spectra in the wavelength range $\sim 3800-9200 \AA$ for SDSS-II galaxies and $\sim 3600-10000 \AA$ for SDSS-III galaxies, and carried out a series of Monte Carlo simulations as described by e.g. Izotov et al. (2015).

The star-formation history is approximated assuming a short burst with age $t$ (burst) $<10$ Myr and a prior continuous star formation with a constant SFR responsible for the older stars, with age starting at $t_{2}=t$ (old) and ending at $t_{1}\left(t_{2}>t_{1}\right.$ and zero time is now). Both $t_{1}$ and $t_{2}$ are varied in Monte Carlo simulations between $10 \mathrm{Myr}$ and 15 Gyr. The best solution of the SED is required to fulfill two conditions. First, only models, in which $\mathrm{EW}(\mathrm{H} \beta)$ agrees with the observed value within $5 \%$, were selected. Second, the best modelled SED among selected models for each set of fixed parameters was found from $\chi^{2}$ minimization of the deviation between the modelled and the observed continua.

The best solutions can be found for different combinations of evolutionary tracks, stellar atmosphere models and initial mass functions with the typical uncertainties of $\sim 0.2$ dex for the stellar mass. In Fig. 5a we show the distribution of burst ages $t$ (burst) with the maximum of $\sim 6$ Myr, corresponding to the equivalent width $\mathrm{EW}(\mathrm{H} \beta) \sim 30 \AA$, while the burst age of 10 Myr corresponds to $\mathrm{EW}(\mathrm{H} \beta) \sim 7 \AA$. The mass fraction of the old stellar population formed continuously with a constant SFR between $t_{2}$ and $t_{1}$ is shown in Fig. 5b with an average value of $\sim 96 \%$ corresponding to the mass ratio of the old-to-young stellar population of $\sim$ 24.

We note that the photometric data were not used in the SED fitting, but they are in general consistent with the SEDs derived from the SDSS optical spectra (e.g. Izotov et al. 2014). In particular, we do not use the near-infrared (NIR) photometric data, which are known only for less than $50 \%$ of galaxies from our sample. It is often assumed that emission of a galaxy in the NIR is produced by the old stellar population. This assumption may not be true for SFGs. The luminosity ratio of the $3 \mathrm{Myr}$ and 10 Gyr instantaneous bursts with equal masses at $10000 \AA$ is $\sim 25$. Therefore, luminosities at this wavelength are equal if the mass of old stellar population is 25 times higher than that of the young stellar population, very similar to the average value in Fig. 5b. This means that, on average, the contribution of the old stellar population in the red part of the SFG SDSS spectrum is considerable, allowing for a reliable mass determination of

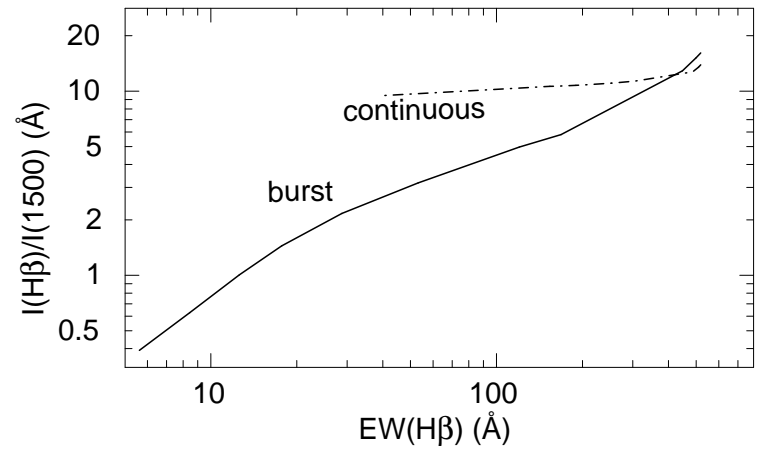

Figure 7. The dependence of the $\mathrm{H} \beta$ to monochromatic $1500 \AA$ flux ratio on the $\mathrm{H} \beta$ equivalent width modelled with the Starburst99 code (Leitherer et al. 1999, 2014). The instantaneous burst and continuous star formation with a constant star formation rate are shown by solid and dash-dotted lines, respectively.

the old population. However, the contribution of the young population is not negligible. The situation is more complex for the SFGs with $\operatorname{EW}(\mathrm{H} \beta)>100 \AA$ because the contribution of the nebular continuum in the visible and NIR ranges is high and it needs to be taken into account (Izotov et al. 2011). On the other hand, the average contribution of the old stellar population to the continuum near the $\mathrm{H} \beta$ emission line is only $\sim 4-5 \%$, indicating that $\operatorname{EW}(\mathrm{H} \beta)$ is a reliable tracer of the starburst age in our compact SFGs.

\section{BURSTING VS CONTINUOUS STAR FORMATION}

In Fig. 6 we show the relations between the FUV-to-H $\beta$ and NUV-to-H $\beta$ luminosity ratios and the stellar masses for the entire sample. We split the sample into objects with high $\mathrm{H} \beta$ equivalent widths $\operatorname{EW}(\mathrm{H} \beta) \geqslant 100 \AA$ (black dots) and with low $\mathrm{H} \beta$ equivalent widths $\mathrm{EW}(\mathrm{H} \beta)<100 \AA$ (grey dots). Clear offsets between the two sets of data are present with ratios, which are on average by $\Delta \sim 0.4$ dex higher for galaxies with low $\mathrm{EW}(\mathrm{H} \beta) \mathrm{s}$.

Dispersions $\sim 0.3-0.4$ dex of galaxies in Fig. 6 are caused in part by uncertainties of the GALEX FUV and NUV magnitudes, which for our faint galaxies are as high as $0.3-0.5 \mathrm{mag}$, corresponding to the FUV and NUV luminosity uncertainties $\sim 0.1-0.2$ dex. Another source of the dispersions in Fig. 6 are uncertainties of the extinction determination from the hydrogen Balmer decrement, which typically correspond to the uncertainties $\sim 0.1-0.2 \mathrm{mag}$ in $A(V)$. These uncertainties translate to the uncertainties $\sim 0.5-1.0 \mathrm{mag}$ in the FUV and NUV ranges, corresponding to uncertainties $\sim 0.2-0.4$ dex in the FUV and NUV luminosities. These two sources of statistical uncertainties are sufficient to explain the observed dispersions in Fig. 6. However, they can not explain the offsets in distributions of SFGs with low and high $\mathrm{EW}(\mathrm{H} \beta) \mathrm{s}$.

One of the possible explanations of these offsets is the bursting nature of star formation in the galaxies in the entire range of stellar masses meaning that star bursts dominate the $\mathrm{H} \beta$ and UV radiation even in the most massive galaxies from our sample. To study this possibility we consider 

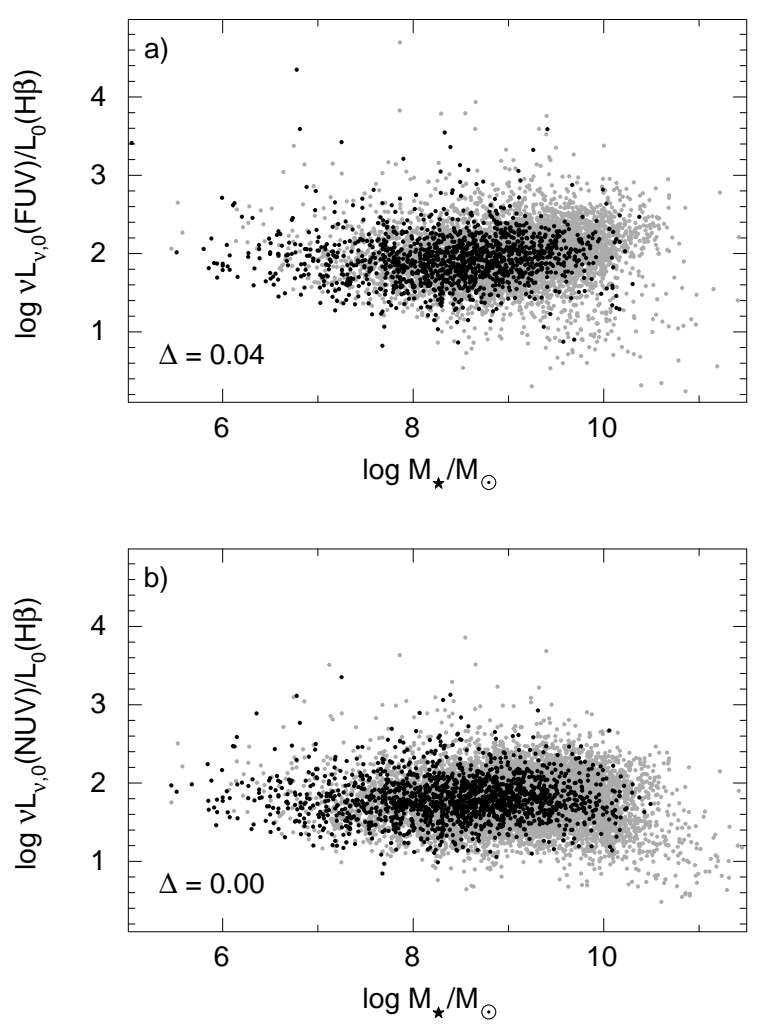

Figure 8. Same as in Fig. 6, but $\mathrm{H} \beta$, FUV and NUV luminosities are reduced to a zero starburst age.

temporal variations of the FUV and $\mathrm{H} \beta$ luminosities for bursting and continuous star formation using Starburst99 models (Leitherer et al. 1999, 2014). In Fig. 7 we show the dependence of the $\mathrm{H} \beta$-to-FUV luminosity ratio on the $\mathrm{H} \beta$ equivalent width $\mathrm{EW}(\mathrm{H} \beta)$ for the instantaneous burst (solid line) and continuous star formation (dash-dotted line). Here we have adopted a Salpeter IMF (Salpeter 1955) with the upper mass limit of $100 \mathrm{M}_{\odot}$ and the low mass limit of $0.1 \mathrm{M}_{\odot}$, Geneva evolutionary tracks (Meynet et al. 1994) of non-rotating stars and a combination of stellar atmosphere models (Lejeune et al. 1997; Schmutz et al. 1992). It is seen that the $\mathrm{H} \beta$-to-FUV luminosity ratio is slowly decreasing with decreasing $\mathrm{EW}(\mathrm{H} \beta)$ in the case of the scenario with continuous star formation while a steep decrease is found for the burst model.

The $\operatorname{EW}(\mathrm{H} \beta)$ is a measure of the age in the instantaneous burst model with the higher value for the younger age. We use $\mathrm{EW}(\mathrm{H} \beta)$ and Starburst99 models to reduce $\mathrm{H} \beta$ luminosity to zero age in the burst model:

$$
\Delta \log L(\mathrm{H} \beta)=2.7-\log [\mathrm{EW}(\mathrm{H} \beta)]
$$

for $\log \mathrm{EW}(\mathrm{H} \beta) \leqslant 2.7$, otherwise $\Delta \log L(\mathrm{H} \beta)=0$.

We note that the age correction is needed not only for the $\mathrm{H} \beta$ luminosity but also for the FUV and NUV luminosities which are produced by young stars. In the bursting model, FUV and NUV luminosities decrease more slowly than the $\mathrm{H} \beta$ luminosity because they are produced by longer-lived stars.

Using the same instantaneous burst models obtained with the Starburst99 code we obtain the following equations for reduction of FUV and NUV luminosities to zero age:

$$
\Delta \log L(\mathrm{FUV})=0.39 \times \Delta \log L(\mathrm{H} \beta),
$$

$$
\Delta \log L(\mathrm{NUV})=0.30 \times \Delta \log L(\mathrm{H} \beta) .
$$

The dependencies of the FUV-to-H $\beta$ and NUV-to-H $\beta$ luminosity ratios on the stellar masses are shown in Fig. 8, where all luminosities are reduced to a zero age. It is seen that no offsets are present between the galaxies with low and high $\mathrm{EW}(\mathrm{H} \beta) \mathrm{s}$. The differences $\Delta$ between average FUV-to$\mathrm{H} \beta$ and NUV-to-H $\beta$ luminosity ratios for galaxies with high and low $\mathrm{EW}(\mathrm{H} \beta) \mathrm{s}$ are $\approx 0$.

Instead, offsets remain in the case of continuous star formation because FUV-to-H $\beta$ and NUV-to- $\mathrm{H} \beta$ luminosity ratios vary little depending on the $\mathrm{EW}(\mathrm{H} \beta)$ or equivalently on the age (dash-dotted line in Fig. 7). Therefore, we conclude that stellar populations producing $\mathrm{H} \beta$ and UV radiation in compact SFGs from our sample are formed during recent short bursts. Of course, we cannot exclude the continuous star formation in our galaxies. However, even if present, its contribution to the galaxy $\mathrm{H} \beta$ emission line and UV continuum luminosities must be very small.

\section{RELATIONS BETWEEN GLOBAL PARAMETERS, CORRECTED FOR THE BURST AGE}

Our finding emphasizes the importance of the age correction in order to compare properties of star formation in similar conditions. Such correction would greatly reduce scatter of points on various diagrams including $\mathrm{H} \beta$ and UV luminosities and SFRs obtained from these luminosities.

\section{1 $\mathrm{H} \beta$ luminosity and star formation rate}

We first consider the relation between the $\mathrm{H} \beta$ luminosity and the stellar mass. It can be transformed to the relation between the SFR and stellar mass by using the Kennicutt (1998) equation.

In Fig. 9a we show the stellar mass $-\mathrm{H} \beta$ luminosity diagram for the galaxies from our sample of compact SFGs in the case when $L(\mathrm{H} \beta)$ is not corrected for the burst age. On the upper abscissa we also show the SFR scale. As expected from the above discussion, a clear offset is present for the galaxies with low and high $\mathrm{EW}(\mathrm{H} \beta) \mathrm{s}$ shown by light-grey and dark-grey dots, respectively, implying that corrections for the starburst age are needed to produce the unbiased relation. For comparison, we show high- $z$ galaxy candidates at $z=9-11$ (Coe et al. 2013; Oesch et al. 2014) (filled circles), which are considered as the ones among the first galaxies formed in the universe, and the low-mass Ly $\alpha$ emitting galaxies at $z=3-6$ (Karman et al. 2016) (filled triangles). It is seen that the location of these high- $z$ galaxies is in good agreement with the location of low- $z$ SFGs from our sample with high $\mathrm{EW}(\mathrm{H} \beta) \geqslant 100 \AA$ (dark-grey dots), indicating similar properties. We note that no correction for the burst age using the $\mathrm{H} \beta$ emission line is possible for the high- $z$ galaxies because $\mathrm{EW}(\mathrm{H} \beta)$ s were not presented by Coe et al. (2013), Oesch et al. (2014) and Karman et al. (2016). 

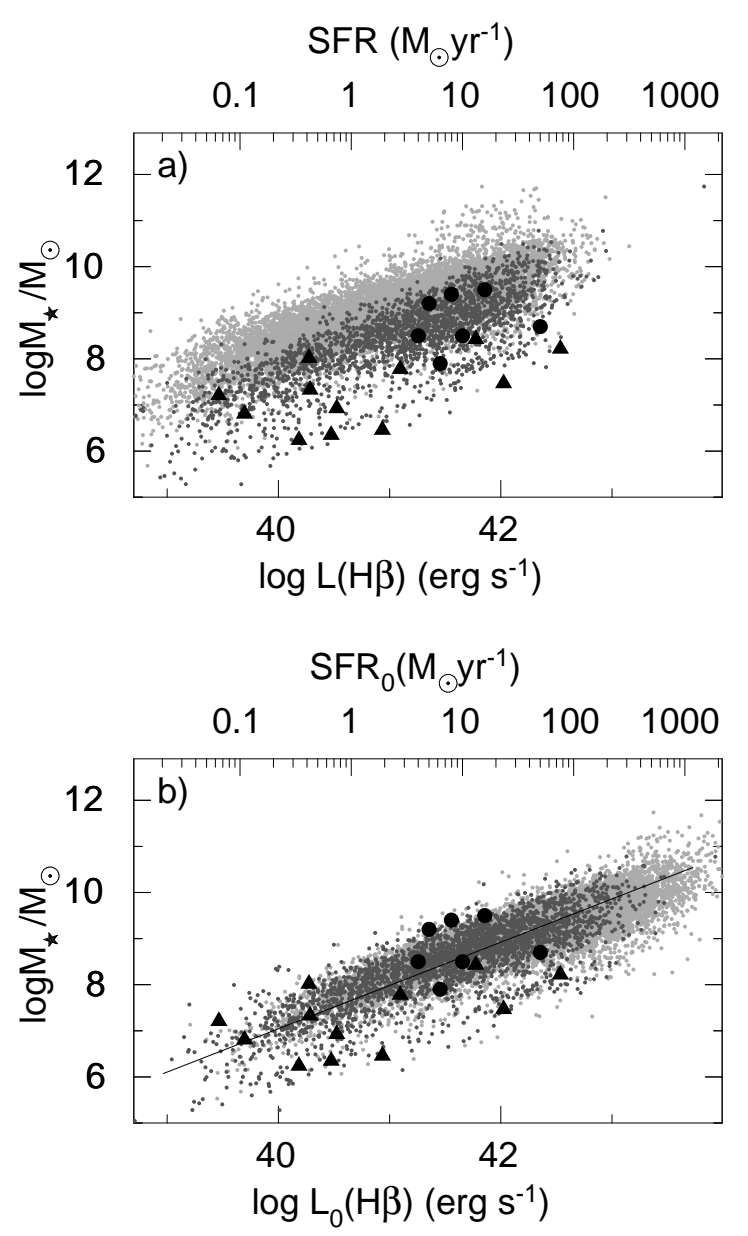

Figure 9. (a) The dependence of the stellar mass $M_{\star}$ on the extinction- and aperture-corrected $\mathrm{H} \beta$ luminosity $L(\mathrm{H} \beta$ ) (lower axis) and star formation rate SFR (upper axis). (b) Same as in (a) but $L(\mathrm{H} \beta)$ and SFR are reduced to zero burst age. In both panels, galaxies with rest-frame $\mathrm{H} \beta$ equivalent widths $\mathrm{EW}(\mathrm{H} \beta)$ $<100 \AA$ and $\operatorname{EW}(\mathrm{H} \beta) \geqslant 100 \AA$ are shown by light-grey and darkgrey dots, respectively. The linear regression in (b) for the entire sample is shown by a black solid line. For comparison, the high-redshift galaxy candidates at $z=9-11$ (Coe et al. 2013; Oesch et al. 2014) and low-mass Ly $\alpha$ emitting galaxies at $z=$ $3-6$ (Karman et al. 2016) are shown by filled circles and filled triangles, respectively.

We show in Fig. 9b the same stellar mass $-\mathrm{H} \beta$ luminosity relation as in Fig. 9a but the $L(\mathrm{H} \beta)$ is reduced to zero age. We denote the corrected $\mathrm{H} \beta$ luminosity as $L_{0}(\mathrm{H} \beta)$ and the respective star formation rate as $\mathrm{SFR}_{0}$. It is seen that no offset is present between the galaxies with low and high $\mathrm{EW}(\mathrm{H} \beta) \mathrm{s}$ and the relation is much tighter as compared to that in Fig. 9a. This relation can be approximated by the dependence:

$$
\frac{M_{\star}}{\mathrm{M}_{\odot}}=3.08 \times 10^{-31} L(\mathrm{H} \beta)^{0.94}=5.06 \times 10^{7} \mathrm{SFR}^{0.94},
$$

where $L(\mathrm{H} \beta)$ is in $\operatorname{erg~s}^{-1}$ and $\mathrm{SFR}$ in $\mathrm{M}_{\odot} \mathrm{yr}^{-1}$. It is very close to a linear dependence suggesting that the SFR is nearly proportional to the stellar mass. We also note that the distribution of low- $z$ compact SFGs is coincident with the distribution of high- $z$ SFGs shown by filled circles and
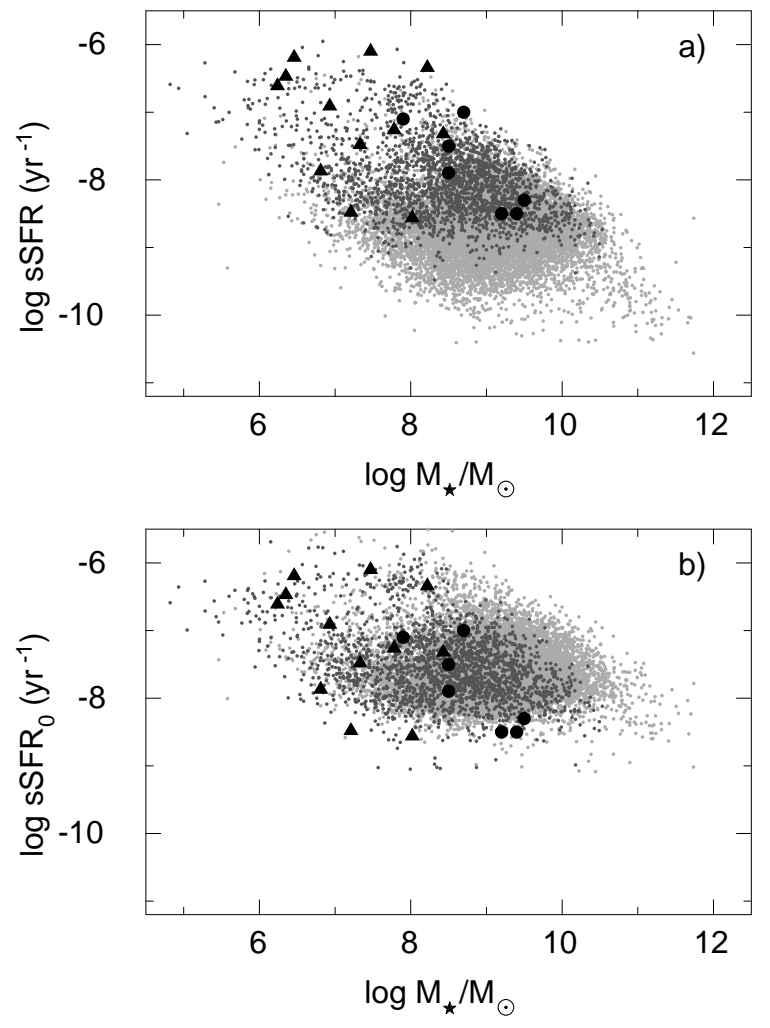

Figure 10. The dependence of the extinction- and aperturecorrected specific star formation rate SSFR on the stellar mass $M_{\star}$. (b) Same as in (a) but sSFR is reduced to zero burst age. In both panels symbols are the same as in Fig. 9.

triangles despite the fact that no age correction is applied for high- $z$ galaxies. However, SFRs for high- $z$ galaxies are derived not from the $\mathrm{H} \beta$ emission line but from the UV continuum luminosities, for which age corrections are much smaller. Furthermore, distributions of low- $z$ SFGs with high $\mathrm{EW}(\mathrm{H} \beta) \mathrm{s}$ and high- $z$ SFGs in Fig. 9a are very similar indicating that the emission of high- $z$ galaxies is also dominated by young bursts. Overall, we assume that burst age corrections for high- $z$ galaxies shown in Fig. 9 should be small.

\subsection{Specific star formation rate}

The offset of the specific star formation rate $\mathrm{sSFR} \equiv$ $\mathrm{SFR} /\left(M_{\star} / \mathrm{M}_{\odot}\right)$ is clearly seen between the galaxies with low and high $\mathrm{EW}(\mathrm{H} \beta) \mathrm{s}$ resulting in a broad distribution of the compact SFGs on the sSFR $-M_{\star}$ diagram (Fig. 10a).

On the other hand, the age correction results in a much narrower distribution (Fig. 10b). This distribution is almost independent on $M_{\star}$, as expected, because the SFR is almost linearly increased with increasing $M_{\star}$ (Eq. 4). We also note that the very high values of $\mathrm{SSFR}_{0}$ of $\sim 10-100 \mathrm{Gyr}^{-1}$, among the highest known so far for galaxies, imply very efficient conversion of the interstellar matter to stars. These values are similar to the ones derived for the highest-redshift galaxies shown in Fig. 10 by filled circles and triangles. However, due to the bursting nature of star formation in our low$z$ compact and high- $z$ SFGs, the specific star formation rate averaged over large time intervals of $1-10$ Gyr would be lower. This averaged value is uncertain because it depends 
both on the time interval adopted for averaging and the unknown number of starbursts during the same interval. It is likely that star formation in our compact galaxies converts interstellar matter to the stellar mass on time scales which may be considerably lower than the cosmological time.

\section{CONCLUSIONS}

In this paper we present a study of the integrated characteristics of a sample of $\sim 14000$ compact star-forming galaxies (SFGs) at redshifts $z \sim 0-1$ selected from the Data Release 12 of the Sloan Digital Sky Survey. Our main results are as follows.

1. We find that the star formation in our galaxies occurs in short strong bursts which dominate the $\mathrm{H} \beta$ and UV continuum radiation while the contribution of the continuous star formation to the $\mathrm{H} \beta$ and $\mathrm{UV}$ luminosities, even if present, is very low.

2. Since the $\mathrm{H} \beta$ and UV luminosities of the compact SFGs are rapidly changing with time, the correction for the starburst age is needed to produce homogeneous sets of data in different samples at various redshifts for their comparison.

3 . We find that the scatter of points in the relation stellar mass $M_{\star}$ - star formation rate (SFR) is greatly reduced if the $\mathrm{H} \beta$ luminosities and SFRs are reduced to zero starburst age. It is shown that SFRs and $L(\mathrm{H} \beta) \mathrm{s}$ are almost linearly increasing with $M_{\star}$. The scatter of specific star formation rates (sSFRs) is also greatly reduced if the correction for the burst age is taken into account. We find that sSFRs for our sample galaxies corrected for the starburst age do not depend on $M_{\star}$, which is a consequence of a nearly linear dependence between $M_{\star}$ and SFR, and are among the highest known for SFGs.

4. It is shown that average stellar masses, $\mathrm{H} \beta$ luminosities, SFRs and sSFRs of our galaxies are similar to the respective characteristics of highest-redshift SFGs and imply that low- $z$ compact SFGs may be considered as local counterparts of the galaxies in the early universe.

\section{ACKNOWLEDGEMENTS}

Funding for the SDSS and SDSS-II was provided by the Alfred P. Sloan Foundation, the Participating Institutions, the National Science Foundation, the U.S. Department of Energy, the National Aeronautics and Space Administration, the Japanese Monbukagakusho, the Max Planck Society, and the Higher Education Funding Council for England. Funding for SDSS-III has been provided by the Alfred P. Sloan Foundation, the Participating Institutions, the National Science Foundation, and the U.S. Department of Energy Office of Science. The SDSS-III web site is http://www.sdss3.org/. SDSS-III is managed by the Astrophysical Research Consortium for the Participating Institutions of the SDSS-III Collaboration. GALEX is a NASA mission managed by the Jet Propulsion Laboratory. This research has made use of the NASA/IPAC Extragalactic Database (NED) which is operated by the Jet Propulsion Laboratory, California Institute of Technology, under contract with the National Aeronautics and Space Administration.

\section{REFERENCES}

Ade P. A. R. et al., 2014, A\&A, 571, A16

Alam S. et al., 2015, ApJS, 219, 12

Baldwin J. A., Phillips M. M., Terlevich R., 1981, PASP, 93,5

Bouwens R. J., Illingworth G. D., Oesch P. A. et al., 2012, ApJ, 754, 82

Cardamone C. et al., 2009, MNRAS, 399, 1191

Cardelli J. A., Clayton G. C., Mathis J. S., 1989, ApJ, 345, 245

Coe D., Zitrin A., Carrasco M. et al., 2013, ApJ, 762, 32

Cullen F., Cirasuolo M., McLure R. J., Dunlop J. S., Bowler R. A. A., 2014, MNRAS, 440, 2300

Izotov Y. I., Thuan T. X., Lipovetsky V. A., 1994, ApJ, 435,647

Izotov Y. I., Guseva N. G., Thuan T. X., 2011, ApJ, 728, 161

Izotov Y. I., Guseva N. G., Fricke K. J., Henkel C., 2014, A\&A, 561, A33

Izotov Y. I., Guseva N. G., Fricke K. J., Henkel C., 2015, MNRAS, 451, 2251

Izotov Y. I., Orlitová I., Schaerer D., Thuan T. X., Verhamme A., Guseva N. G., Worseck G., 2016a, Nature, 529, 178

Izotov Y. I., Schaerer D., Thuan T. X., Worseck G., Guseva N. G., Orlitová I., Verhamme A., 2016b, MNRAS, 461, 3683

Karman W. et al., 2016, preprint (arXiv:1606.01471)

Kauffmann G. et al., 2003, MNRAS, 341, 33

Kennicutt R. C., Jr., 1998, Ann.Rev.Astron.Astrophys., 36, 189

Leitherer C. et al., 1999, ApJS, 123, 3

Leitherer C., Ekström S., Meynet G., Schaerer D., Agienko

K. B., Levesque E. M., 2014, ApJS, 212, 14

Lejeune T., Buser R., Cuisinier F., 1997, A\&AS, 125, 229

Maier C., Lilly S. J., Ziegler B. L., Pérez Montero E., Peng

Y., Balestra I., 2014, ApJ, 792, 3

Maiolino R. et al., 2008, A\&A, 488, 463

Manucci F., Cresci G., Maiolino R., Marconi A., Gnerucci A., 2010, MNRAS, 408, 2115

Meynet G., Maeder A., Schaller G., Schaerer D., Charbonnel C., 1994, A\&AS, 103, 97

Oesch P. A., Bouwens R. J., Illingworth G. D. et al., 2014, ApJ, 786, 108

Salmon B., Papovich C., Finkelstein S. L. et al., 2015, ApJ, 799, 183

Salpeter E. E., 1955, ApJ, 121, 161

Sanders R. L., Shapley A. E., Kriek M. et al., 2015, ApJ, 799,138

Schmutz W., Leitherer C., Gruenwald, R., 1992, PASP, 104, 1164

Steidel C. S. et al., 2014, ApJ, 795, 165

Tremonti C. et al., 2004, ApJ, 613, 898

Troncoso P. et al., 2014, A\&A, 563, A58

Wright E. L., 2006, PASP, 118, 1711

This paper has been typeset from a $\mathrm{T}_{\mathrm{EX}} \mathrm{X} \mathrm{LT}_{\mathrm{E}} \mathrm{X}$ file prepared by the author. 